Gloria Lliso Ribera: None declared, Alessandra Nerviani: None declared, Rebecca Hands: None declared, Giovanni Giorli: None declared, Barbara Frias: None declared, Edyta Jaworska: None declared, Katriona Goldmann: None declared, Myles Lewis Grant/research support from: Celgene, Antonio Manzo: None declared, Michele Bombardieri Grant/research support from: Celgene, Consultant for: Medimmune, Costantino Pitzalis Grant/ research support from: Celgene

DOI: 10.1136/annrheumdis-2019-eular.1284

\section{THU0059 INCIDENCE OF JOINT REPLACEMENT SURGERY AMONG BIOLOGICS AND NON-BIOLOGICS TREATED PATIENTS WITH RHEUMATOID ARTHRITIS: A PROPENSITY SCORE MATCHED COHORT STUDY FROM DENMARK}

René Cordtz ${ }^{1,2}$, Samuel Hawley ${ }^{3}$, Daniel Prieto-Alhambra ${ }^{3}$, Lars Erik Kristensen ${ }^{2}$, Søren Overgaard ${ }^{4}$, Anders Odgaard ${ }^{5}$, Lene Dreyer ${ }^{6,7} .{ }^{1}$ Rigshospitalet-Gentofte, Center for Rheumatology and Spine Diseases, Copenhagen, Denmark; ${ }^{2}$ Bispebjerg and Frederiksberg Hospital, The Parker Institute, Copenhagen, Denmark; ${ }^{3}$ University of Oxford, NDORMS, Oxford, United Kingdom; ${ }^{4}$ Odense University Hospital, Department of Orthopaedic Surgery and Traumatology, Odense, Denmark; ${ }^{5}$ Herlev and Gentofte University Hospital, Department of Orthopaedic Surgery, Copenhagen, Denmark; ${ }^{6}$ Aalborg University and Aalborg University Hospital, Dept. of rheumatology, Aalborg, Denmark; ${ }^{7} D A N B I O$, nationwide, Denmark

Background: Biologics have improved several clinical, patient-reported and radiological outcomes in patients with rheumatoid arthritis (RA), but little is known about the potential impact on the need for joint replacement surgery.

Objectives: To investigate the incidence of joint replacement surgery among biologics treated compared with biologics naïve patients with RA.

Methods: A nationwide, register-based propensity score matched cohort study. RA patients registered between 2006 and 2016 in the DANBIO register with a disease duration $\leq 2$ years were identified. Patients initiating their first treatment series with biologics were followed up to 10 years for a first joint replacement of the hip, knee, shoulder, elbow and finger/ wrist. Biologics naïve patients were followed up for the same outcome from their first clinical visit registered in DANBIO. Following a 1 :n propensity score matching, Cox-models were undertaken to calculate the hazard ratio $(\mathrm{HR})$ for a first joint replacement surgery among biologics compared with non-biologics treated RA patients. Further, subgroup analyses based on within-strata propensity score matched patients were carried out. All information on surgical outcomes was obtained in the Danish National Patient Registry.

Results: In total, 1187 biologics treated were matched with 3666 non-biologics treated patients (See Table).

Abstract THU0059 - Table1. Baseline characteristics of biologics treated and biologics naïve patients with rheumatoid arthritis and a disease duration $<2$ years registered in DANBIO between 2006 and 2016.

\begin{tabular}{lccc}
\hline & $\begin{array}{c}\text { Biologics } \\
\text { treated }\end{array}$ & $\begin{array}{c}\text { Biologics } \\
\text { naive }\end{array}$ & SMD \\
\hline N Total & 1187 & 3666 & \\
Female, $\mathbf{n}(\%)$ & $828(70)$ & $2546(69)$ & 0.01 \\
Age at start of follow-up, mean (s.d) & $53.8(13.5)$ & $54.1(15.1)$ & 0.02 \\
Age at diagnosis, mean (s.d) & $52.7(13.5)$ & $52.9(15.1)$ & 0.02 \\
IgM-RF and/or ACPA positive at start of & $686(58)$ & $2042(56)$ & 0.04 \\
follow-up, $\mathbf{n}$ (\%) & & & \\
DAS28-CRP & $4.5[3.6$ to 5.4] & $4.5[3.4$ to 5.4] & 0.03 \\
HAQ-DI & $1.00[0.50$ to & $1.00[0.50$ to & 0.03 \\
& $1.62]$ & $1.62]$ & \\
CRP mg/ml & $9[3$ to 22] & $9[3$ to 20] & 0.02 \\
VAS physician global & $32[18$ to 49] & $30[16$ to 50$]$ & 0.04 \\
VAS pain & $53[31$ to 73] & $52[30$ to 74] & 0.01 \\
Methotrexate, $\mathbf{n}$ (\%) & $1044(88)$ & $3160(86)$ & 0.06 \\
Glucocorticoid, $\mathbf{n}$ (\%) & $246(21)$ & $718(20)$ & 0.04 \\
Chronic obstructive pulmonary disease, $\mathbf{n}(\%)$ & $168(5)$ & $57(5)$ & 0.01 \\
Cardiovascular disease, $\mathbf{n}$ (\%) & $322(9)$ & $97(8)$ & 0.02 \\
Hospitalised with infection in previous $\mathbf{5}$ & $128(4)$ & $43(4)$ & 0.01 \\
years, $\mathbf{n}$ (\%) & & & \\
\hline
\end{tabular}

Abbreviations: SMD, absolute standardized mean difference; s.d., standard deviation; VAS, visual analogue scale. All values are median [interquartile range] unless otherwise stated. During follow-up, 43 biologics-treated and 124 naive patients had a first joint replacement surgery corresponding to an overall HR of 0.95 (0.65 to 1.40) for biologics treated compared with naïve patients (Figure 1). Patients with a DAS28-CRP $<4.6$ at start of follow-up (low and moderate disease activity $)^{1}$, biologics treated had a lower risk of joint replacements compared with biologics naïve patients.
Conclusion: In this nationwide Danish cohort study, there was no difference in the incidence of joint replacement surgery among newly diagnosed RA patients selected for treatment with biologics compared with patients naïve to biologics.

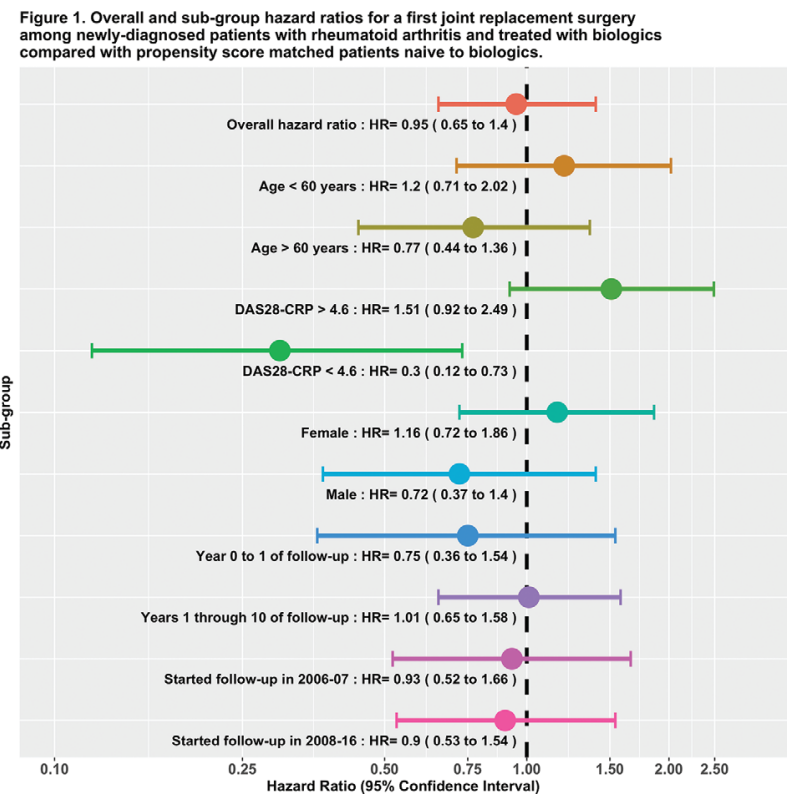

Abstract THU0059 - Figure 1

\section{lREFERENCES:}

[1] Fleischmann, et al. DAS28-CRP and DAS28-ESR cut-offs for high disease activity in rheumatoid arthritis are not interchangeable. RMD Open 2017;3:2-6.

Disclosure of Interests: René Cordtz: None declared, Samuel Hawley: None declared, Daniel Prieto-Alhambra Grant/research support from: Grants from Amgen, UCB Biopharma and Servier outside the submitted work, Consultant for: UCB Biopharma, Speakers bureau: Amgen, Lars Erik Kristensen Grant/research support from: UCB, Biogen, Janssen Pharmaceuticals, and Novartis, Consultant for: Consultant for AbbVie, Amgen, Biogen, BMS, Celgene, Eli Lilly, Janssen Pharmaceuticals, MSD, Novartis, Pfizer, Roche, Sanofi, and UCB Pharma., Speakers bureau: Pfizer, AbbVie, Amgen, UCB, BMS, Biogen, MSD, Novartis, Eli Lilly and Company, and Janssen Pharmaceuticals, Søren Overgaard: None declared, Anders Odgaard: None declared, Lene Dreyer Consultant for: MSD, UCB and Janssen Pharmaceuticals, Speakers bureau: MSD, UCB and Janssen Pharmaceuticals, Speakers bureau: UCB, MSD, Eli Lilly and Janssen Pharmaceuticals.

DOI: 10.1136/annrheumdis-2019-eular.991

\section{THU0060 ELEVATED SYNOVIAL MUC1 EXPRESSION IS ASSOCIATED WITH JOINT DAMAGE AND ONE-YEAR RADIOGRAPHIC PROGRESSION IN RHEUMATOID ARTHRITIS}

Yaoyao Zou ${ }^{1}$, Jun Jing ${ }^{1}$, Jiale Qin², Feng Zhou², Ting Liu², Jun-Wei Wang ${ }^{1}$, Jianzi Lin', Lie Dai'. ' 'Sun Yat-Sen Memorial Hospital, Sun Yat-Sen University, Department of Rheumatology, Guangzhou, China; ${ }^{2}$ Sun Yat-Sen University, Zhong Shan School of Medicine, Guangzhou, China

Background: The tumor-like transformation of synovium, especially the lining layer, play a critical role in joint damage in rheumatoid arthritis (RA) by forming aggressive and invasive rheumatoid pannus, but the underlying mechanisms remains unknown. Mucin1(MUC1), an O-glycosylated protein participating in forming protective mucous barriers on epithelial surfaces and intracellular signaling, has been found aberrantly expressed in many cancers and implicated in tumor progression and metastasis. However, the expression of MUC1 in synovium and its role in RA is unclear.

Objectives: We aimed to investigate the expression of MUC1 in RA synovium and its correlation with joint destruction in RA patients. 